3. J. Dieudonné, La dualite dans les espaces rectoriels topologiques, Ann. Ecole Norm. (3) vol. 59 (1942) pp. 107-139.

4. G. Köthe, Die Quotientenrailme eines linearen vollkommenen Raumes, Math. Zeit. vol. 51 (1947) pp. 17-35.

5. M. Krein and V. Smulian, On regularly convex sets in the space conjugate to a Banach space, Ann. of Math. vol. 41 (1940) pp. 556-583.

6. G. W. Mackey, On infinite dimensional spaces, Trans. Amer. Math. Soc. vol. 57 (1945) pp. 155-207.

7. M. E. Munroe, Homomorphisms on Banach spaces, Bull. Amer. Math. Soc. vol. 54 (1948) pp. 776-781.

UNIVERSITY OF NANCY

\title{
A PROOF THAT THE GROUP OF ALL HOMEO- MORPHISMS OF THE PLANE ONTO ITSELF IS LOCALLY ARCWISE CONNECTED
}

M. K. FORT, JR.

Let $P$ be a plane and let $H(P)$ be the group of all homeomorphisms of the plane $P$ onto itself. We topologize $H(P)$ by defining convergence to mean uniform convergence on each compact subset of $P$. The resulting topology is equivalent to the compact-open topology defined in [1] $]^{1}$ by Fox. It is also known (see [4]) that $H(P)$ is a topological group under this topology. The result obtained in this paper is the following theorem.

Theorem. $H(P)$ is locally arcwise connected.

1. A metric for $H(P)$. We assume a rectangular coordinate system for $P$ and let $d$ be the corresponding metric for $P$. For each positive number $r$ we define $S(r)$ to be the set of all points $(u, v)$ in $P$ such that $\max (|u|,|v|) \leqq r$. If $f$ and $g$ are members of $H(P)$ we define

$$
\rho(f, g)=\sup _{r>0} \min \left(1 / r, \sup _{x \in S(r)} d(f(x), g(x))\right) .
$$

It is a routine matter to verify that $\rho$ is a distance function which defines an admissible metric for $H(P)$. A metric which is essentially the same as $\rho$ is used by M. Bebutoff in [2]. We shall make use of the fact that $\rho(f, g)<\epsilon$ if and only if $d(f(x), g(x))<\epsilon$ for all $x$ in $S(1 / \epsilon)$.

2. Isotopy and arcs. By an isotopy we shall mean a homotopy

Presented to the Society, November 27, 1948; received by the editors October 9, 1948.

${ }^{1}$ Numbers in brackets refer to the bibliography at the end of the paper. 
$F$ such that $F_{t} \in H(P)$ whenever $0 \leqq t \leqq 1$. It follows from a theorem of Fox in [1] that an isotopy is equivalent to a continuous function on the unit interval into $H(P)$. Since the image of the unit interval under a continuous function is always arcwise connected, we see that members $f$ and $g$ of $H(P)$ can be joined by an arc in a subset $U$ of $H(P)$ if and only if there exists an isotopy $F$ such that $F_{0}=f, F_{1}=g$, and $F_{t} \in U$ whenever $0 \leqq t \leqq 1$.

3. Proof of the theorem. Since $H(P)$ is a topological group, it is sufficient to prove that $H(P)$ is locally arcwise connected at the identity homeomorphism $I$. To do this it is sufficient to show that corresponding to each positive number $\epsilon$ there is a positive number $\delta$ such that if $\rho(f, I)<\delta$, where $f \in H(P)$, then there exists an isotopy $B$ such that $B_{0}=f, B_{1}=I$, and $\rho\left(B_{t}, I\right)<\epsilon$ if $0 \leqq t \leqq 1$.

Suppose $\epsilon>0$. We may assume without loss of generality that $\epsilon<1$. Choose a positive number $\delta$ such that $\delta<\epsilon / 7$ and such that $2 / \delta$ $=(2 n+2) \delta$ for some positive integer $n$. It is well known that $H(P)$ contains exactly two components, the component containing $I$ consisting of all orientation preserving homeomorphisms. In this connection see [8]. We shall also assume $\delta$-small enough so that the $\delta$-neighborhood of $I$ contains only orientation preserving homeomorphisms. Now choose any $f$ such that $f \in H(P)$ and $\rho(f, I)<\delta$.

We define $T$ to be the isotopy for which $T_{t}, 0 \leqq t \leqq 1$, is the translation which increases the first coordinate of each point of $P$ by $t(2 / \delta+\delta)$ and leaves the second coordinate invariant. Define $C_{t}$, $0 \leqq t \leqq 1$, to be the set $T_{t}(S(1 / \epsilon))$, and then define $K$ to be the set $\mathrm{U}_{0 \leqq t \leqq 1} C_{t}$. Finally, we define $R_{0}=S(1 / \delta)$ and $R_{1}=T_{1}\left(R_{0}\right)$.

It will be convenient to prove two lemmas.

Lemma 1. There exists $h \in H(P)$ such that $h\left|C_{0}=f\right| C_{0}, h\left|C_{1}=I\right| C_{1}$ and $d(x, h(x))<\epsilon$ for all $x$ in $K$.

Proof. Let the segment $\overline{a b}$ be the side of the square $R_{1}$ consisting of all points of $R_{1}$ whose first coordinate is a minimum and let $\overline{\alpha \beta}$ be the side of $R_{0}$ consisting of all points of $R_{0}$ whose first coordinate is a maximum. We may assume that $a$ and $\alpha$ have the same second coordinate. Define $x_{0}$ to be the point on $\overline{a b}$ which is at distance $\delta$ from $a$. If $x_{i}, 0 \leqq i<n$, has been defined, we then define $x_{i+1}$ to be the point on $\overline{a b}$ which is at distance $2 \delta$ from $x_{i}$ and which is between $x_{i}$ and $b$. Since $\overline{a b}$ is of length $2 / \delta$ and $2 / \delta=(2 n+2) \delta, x_{n}$ is at distance $\delta$ from $b$.

We define $y_{i}, 0 \leqq i \leqq n$, to be the point of $f\left(R_{0}\right)$ nearest to $x_{i}$ which has the same second coordinate as $x_{i}$. Now define $z_{i}, 0 \leqq i \leqq n$, to be 
the point $f^{-1}\left(y_{i}\right)$. Since $f$ transforms each point of $R_{0}$ a distance less than $\delta$, it is easy to see that the points $z_{i}$ all lie on $\overline{\alpha \beta}$ and moreover lie in the same order on $\overline{\alpha \beta}$ as the corresponding points $x_{i}$ lie on $\overline{a b}$.

We now define $h^{*}$ to be the homeomorphism which agrees with $f$ on $R_{0}$, which agrees with $I$ on $R_{1}$, and which transforms each segment $\overline{z_{i} x_{i}}, 0 \leqq i \leqq n$, linearly into the segment $\overline{y_{i} x_{i}}$. We now make use of a theorem of Gehman (see [3]) and extend $h^{*}$ to a homeomorphism $h$ of $P$ onto $P$.

Let $Q_{i}, 0 \leqq i<n$, be the trapezoid (with interior) which has vertices $x_{i}, x_{i+1}, z_{i+1}, z_{i}$. It is easily seen that for each $i, 0 \leqq i<n$, there is a rectangle with sides of length $6 \delta$ and $2 \delta$ which contains both $Q_{i}$ and $h\left(Q_{i}\right)$. Thus, if $x \in Q_{i}$ we obtain $d(x, h(x)) \leqq \delta(40)^{1 / 2}<7 \delta=\epsilon$. It follows that if $x \in R_{0} \cup R_{1} \cup \cup_{i=0}^{n-1} Q_{i}$ then $d(x, h(x))<\epsilon$. Moreover, it is easily seen that $K \subset R_{0} \cup R_{1} \cup \cup_{i=0}^{n-1} Q_{i}$. We therefore see that the homeomorphism $h$ has the desired properties.

LemMA 2. There exists an isotopy $G$ such that $G_{0}=f, G_{1}\left|C_{0}=I\right| C_{0}$ and $\rho\left(G_{t}, I\right)<\epsilon$ whenever $0 \leqq t \leqq 1$.

Proof. Let $h$ be as in Lemma 1. We then define $G_{t}=T_{t}^{-1} h T_{t} h^{-1} f$ whenever $0 \leqq t \leqq 1$. This clearly defines an isotopy $G$. Since $T_{0}=I$, we readily obtain $G_{0}=f$.

Let $x \in C_{0}$. We obtain $h^{-1} f(x)=x$ since $h\left|C_{0}=f\right| C_{0}$. Now, using the fact that $T_{1}(x) \in C_{1}$ and $h\left|C_{1}=I\right| C_{1}$, we obtain $h T_{1} h^{-1} f(x)$ $=h T_{1}(x)=T_{1}(x)$. Therefore

$$
G_{1}(x)=T_{1}^{-1} h T_{1} h^{-1} f(x)=T_{1}^{-1} T_{1}(x)=x .
$$

We have shown that $G_{1}\left|C_{0}=I\right| C_{0}$.

Suppose $0 \leqq t \leqq 1$ and $x \in C_{0}$. Then $T_{t} h^{-1} f(x)=T_{t}(x) \in K$. Thus $h T_{t} h^{-1} f(x)$ is within $\epsilon$ of $T_{t}(x)$. Since $T_{t}$ is a translation, this fact implies that $T_{t}^{-1} h T_{t} h^{-1} f(x)$ is within $\epsilon$ of $T_{t}^{-1} T_{t}(x)=x$. Thus $d\left(G_{t}(x), x\right)$ $<\epsilon$ for each $x \in C_{0}=S(1 / \epsilon)$ whenever $0 \leqq t \leqq 1$. We therefore obtain $\rho\left(G_{t}, I\right)<\epsilon$ whenever $0 \leqq t \leqq 1$.

We now use the isotopy $G$ defined in Lemma 2 to define the isotopy $B$. If $x \in P$ we define:

$$
\begin{array}{ll}
B_{t}(x)=G_{2 t}(x) & \text { for } 0 \leqq t \leqq 1 / 2 ; \\
B_{t}(x)=G_{1}(2(1-t) x) / 2(1-t) & \text { for } 1 / 2<t<1 ;
\end{array}
$$

and

$$
B_{1}(x)=I(x) \text {. }
$$

It is easily verified that $B$ has the desired properties. 


\section{BIBLIOGRAPHY}

1. R. H. Fox, On topologics for function spaces, Bull. Amer. Math. Soc. vol. 51 (1945) pp. 429-432.

2. M. Bebutoff, Sur les systèmes dynamiques dans l'espace des fonctions continues, Akademia Nauk, Doklady vol. 27 (1940) pp. 904-906.

3. H. M. Gehman, On extending a continuous (1-1) correspondence of two plane continuous curves to a correspondence of their planes, Trans. Amer. Math. Soc. vol. 28 (1926) pp. 252-265.

4. R. F. Arens, Topologies for homeomorphism groups, Amer. J. Math. vol. 68 (1946) pp. 593-610.

5. G. Birkhoff, Topology of transformation sets, Ann. of Math. vol. 35 (1934) pp. 861-875.

6. J. Schreier and S. Ulam, Über topologische Abbildungen der euklidischen Sphären, Fund. Math. vol. 23 (1934) pp. 102-118.

7. J. W. Alexander, On the deformation of $n$-cell, Proc. Nat. Acad. Sci. U. S. A. vol. 9 (1923) pp. 406-407.

8. J. H. Roberts, Concerning homeomorphisms of the plane into itself, Bull. Amer. Math. Soc. Abstract 44-9-402.

UNIVERSITY OF VIRGINIA and UNIVERSITY OF ILLINOIS 